\title{
PRAGUE CASTLE AREA LOCAL STABILITY DETERMINATION ASSESSMENT BY THE ROBUST TRANSFORMATION METHOD
}

\author{
Martin ŠTRONER ${ }^{1)}$ *, Rudolf URBAN ${ }^{1)}$, Pavel RYS ${ }^{1)}$ and Jan BALEK ${ }^{2)}$ \\ 1) Department of the special geodesy, Faculty of the Civil Engineering, Czech Technical University in Prague, \\ Thákurova 7, Prague 6, Czech Republic \\ ${ }^{2)}$ Institute of Rock Structure and Mechanics of the ASCR, v.v.i., V Holešovičkách 41, 18209 Prague 8, Czech Republic \\ *Corresponding author's e-mail: martin.stroner@fsv.cvut.cz
}

\begin{tabular}{l} 
ARTICLE INFO \\
\hline Article history: \\
Received 13 November 2014 \\
Accepted 3 December 2014 \\
Available online 16 December 2014 \\
\hline
\end{tabular}

Keywords:

Robust estimates

Least squares adjustments

Deformations measurement

\section{ABSTRACT}

A geodetic measurement of shifts and deformations is a well-known method. In the paper there is presented a methodology of the shifts assessment based on the robust estimation calculation and the following statistical testing. The methodology is exceptional in the fact, that every point can be potentially unstable and selection of the stable points is a part of the calculation process. No undoubtedly stable points are needed. Procedure was applied to a geodetic network of Prague Castle.

\section{INTRODUCTION}

A geodetic measurement of shifts and deformations is a well-known method. The methodology, attributes and possibilities of this method are described in many publications. It is possible to present the measurement of shifts and reshaping of construction (Rueger, 2006), their parts (Procházka et al., 2010) or complex engineering facilities (Teskey et al., 1996), geological structures (Colesanti and Wasovsky, 2006) and the whole geological areas (Švábenský et al., 2012) or (Darby and Williams, 1991). Usually the terrestrial methods are chosen for the measurement. In case of heights it is best to use the precision leveling, for a position the geodetic networks and the link traverses are used, and of course the methods of Global Navigation Satellite Systems (GNSS e.g. in (Gokalp and Tasci, 2009)), photogrammetry (e.g. (Pavelka and Řezníček, 2010) or (Hamouz et al., 2014)), 3D laser scanning (e.g. (Dunning et al., 2009) or (Koska et al., 2008)) and Synthetic Aperture Radar interferometry (SAR, e.g. in (Colesanti and Wasovsky, 2006)). With all these methods it is necessary to create the measurement accuracy project of the shifts as is required in standards, e.g. in CSN 730405. This subject is also described in (Sabová and Pukanská, 2007). Also other non-geodetics methods are used for measurements. The geotechnical methods (Procházka et al., 2010) are usually used in a smaller areas for measurements of inclinations and deformations of individual building or landscape elements. The key parts in the measurements are geotechnical boreholes which are described also in (Procházka et al., 2010). These boreholes are used not only for monitoring of the
Earth surface, but also for monitoring of shifts under the surface. There may also be used the ground penetrating radars (Hruška and Hubatka, 2000).

The fundamental parts of the whole common geodetic methods are the knowledge and the accessibility of points that are stable throughout the continual or epoch measurements. When all points can be affected by changes, it is difficult to make conclusions on the shift of individual observed points. This situation may arise, for example, if the stable points are located too far from the measured points and determination of the shifts is not therefore accurate. In this case it is suitable to use a process of evaluation presented in this article. First it is necessary to determine probably shifting points. The basic tool for evaluation can be a robust linear transformation (calculation of the transformation key by the robust adjustment).

At first the principle of used robust methods will be explained (for this article only m-estimates), further their application to a linear transformation and the total processing procedure of the stage measurements. The procedure was applied to a geodetic network of Prague Castle in two different types of measurement (first for a height network and second for a positioning network). The process was divided in two, because the measurements were done separately too. The height measurement was done by a precise levelling and the position was done by a trigonometric measurement of angles and distances by a total station. The presented examples have one more special feature: Monitoring was carried out on the basis of annual requirements by the management of the Prague Castle and unfortunately the set of the 
observed points was not the same in each epoch, but even this uncommon results of measurement can be evaluated using this methods.

\section{PRINCIPLE OF THE ROBUST ADJUSTMENT}

The robust adjustment used for the transformation key calculation is the key of the method, outliers (here possible shifts) are there more reliably identified than in case of use of the least squares method (LSM). From the point of view of the adjustment the methods of the robust estimate are much more resistant against the outliers' presence (here shifted points). The robust methods are described in detail in the fundamental article (Huber, 1964), in overview in (Huber, 1981), (Koch, 1999) and (Hampel et al., 1986), and in (Štroner and Hampacher, 2011) with extensive list of the individual methods. Precisely is the calculation procedure described in (Třasák and Štroner, 2014) together with the testing of the outlier detection efficiency in geodetic networks. Useful methods of the robust adjustment are based on the maximum likelihood method.

According to (Koch, 1999) it is a solution to the minimization problem. The base is formed by the requirement for the maximum likelihood solution given by expression:

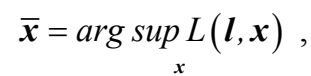

Where $\boldsymbol{l}$ is the random vector (measurement), $L(\boldsymbol{l}, \boldsymbol{x})$ the likelihood (probability) function, $\overline{\boldsymbol{x}}$ estimate parameters (unknowns). In other words, the solution has to be the most probable one. If the random vector of an observation $\boldsymbol{l}$ has a density of probability $f(\boldsymbol{x})$, which depends on the fixed and unknown parameters $\boldsymbol{x}$, then the likelihood function is:

$$
L(\boldsymbol{l}, \boldsymbol{x})=f(\boldsymbol{x}) .
$$

The linear model of the problem is:

$$
A \boldsymbol{x}=\boldsymbol{l}+\boldsymbol{\varepsilon},
$$

$$
\boldsymbol{A}=\left(\begin{array}{c}
\boldsymbol{a}_{1}^{T} \\
\boldsymbol{a}_{2}^{T} \\
\vdots \\
\boldsymbol{a}_{n}^{T}
\end{array}\right), \quad \boldsymbol{x}=\left(\begin{array}{c}
x_{1} \\
x_{2} \\
\vdots \\
x_{u}
\end{array}\right),
$$

where $\boldsymbol{x}$ is the vector of unknowns, $\boldsymbol{l}$ is the vector of observations (measurements), $\boldsymbol{\varepsilon}$ is the vector of real error, $\boldsymbol{A}$ is the matrix of linear (linearized) relationships between measurements and unknown parameters. The number of measurements $n$ is larger than the number of unknowns $u$. It is a calculation with adjustment. The measurements are independent. The equation of the observation for measurements $l_{i}$ :

$l_{i}+\varepsilon_{i}=\boldsymbol{a}_{i}^{T} \boldsymbol{x}$.
The unknowns in the vector $\boldsymbol{x}$ are determined by the method of maximum likelihood, provided that the density of the probability $p\left(l_{i}\right)$ observation $l_{i}$ is directly proportional to the function $f\left(\boldsymbol{l}_{\boldsymbol{i}}, \boldsymbol{x}\right)$, i.e. the following applies:

$p\left(l_{i}\right) \propto f\left(l_{i}, x\right)$.

The density of the probability $p(l)$ for an independent measurement is then:

$p(\boldsymbol{l}) \propto \prod_{i=1}^{n} f\left(l_{i}, \boldsymbol{x}\right)=f\left(l_{1}, \boldsymbol{x}\right) \cdot f\left(l_{2}, \boldsymbol{x}\right) \cdots f\left(l_{n}, \boldsymbol{x}\right)$

A prerequisite for the method of maximum likelihood is the knowledge of the probability distribution. With regard to the central limit theorem it is further assumed that the measurements have normal distribution $N\left(\boldsymbol{A} \boldsymbol{x}, \sigma^{2} \boldsymbol{I}\right)$. Then, for the likelihood function, applies:

$L\left(\boldsymbol{l} ; \boldsymbol{x}, \sigma^{2}\right)=\frac{1}{\left(2 \pi \sigma^{2}\right)^{n / 2}} e^{\frac{-\varepsilon^{T} \varepsilon}{2 \sigma^{2}}}$.

The task is to maximize the likelihood (probability), the problem is solved by differentiation of the unknowns $x$ and standard deviations $\sigma^{2}$ and obtained by placing the expressions to zero. Due to the characteristic function of the normal distribution and the likelihood function it is advantageous to derive the logarithm of this function:

$\ln L\left(\boldsymbol{l} ; \boldsymbol{x}, \sigma^{2}\right)=-\frac{n}{2} \ln (2 \pi)-\frac{n}{2} \sigma^{2}-\frac{1}{2 \sigma^{2}} \varepsilon^{T} \varepsilon$.

(After the derivation according to the unknowns $\boldsymbol{x}$, the result is an estimation of the least squares method). The expression is therefore maximized

$\prod_{i=1}^{n} f\left(l_{i}, \boldsymbol{x}\right)$ minimized

Or, using the previous logarithmic formulas, $\sum_{i=1}^{n}\left(-\ln f\left(l_{i}, \boldsymbol{x}\right)\right)$

which leads to the least squares method. This function of estimation is given by the assumption of a normal distribution of the measurement and must be replaced by a more suitable function, because the normal distribution prerequisite is not accomplished. The calculation of robust M-estimators (Huber, 1964) is determined by minimizing the expression:

$\sum_{i=1}^{n} \rho\left(l_{i}, g_{i}(\boldsymbol{x})\right)=\min$.

where $\rho$ is a suitable estimator function (,score function"), $g_{i}(\boldsymbol{x})$ is a function of unknowns parameters. The estimator is not constant as in the 
case of the least squares method. The derivation of the estimator (i.e. the influential function) is:

$$
\psi\left(l_{i}, \boldsymbol{x}\right)=\frac{\partial}{\partial g_{i}(\boldsymbol{x})} \rho\left(l_{i}, g_{i}(\boldsymbol{x})\right)
$$

The $\overline{\boldsymbol{x}}$ estimate of an unknown parameters $\boldsymbol{x}$ :

$$
\begin{aligned}
& \sum_{i=1}^{n} \frac{\partial \rho\left(l_{i}, g_{i}(\boldsymbol{x})\right)}{\partial \hat{x}_{k}}=\sum_{i=1}^{n} \psi\left(l_{i}, \hat{\boldsymbol{x}}\right) \frac{\partial g_{i}(\hat{\boldsymbol{x}})}{\partial \hat{x}_{k}}=0 \\
& \quad \text { for } k \in\{1, \ldots, u\} .
\end{aligned}
$$

The robust estimate can be found using $\psi\left(l_{i}, \overline{\boldsymbol{x}}\right)$. Only when the function $\psi\left(l_{i}, \overline{\boldsymbol{x}}\right)$ is bounded it is the robust estimate, because it is proportional to the influence function (Hampel et al., 1986). The influence function describes the effect of further observations to the estimate. Along with the breakdown point, these are important characteristics describing specific robust estimate. The breakdown point is the smallest proportion of observations that, after replacing by any values, can lead to incorrect values of the estimate (see (Hampel et al., 1986)).

For routine calculations it is appropriate to introduce the concept of a standard error, i.e. the proportion of errors and the corresponding standard deviation of a measurement:

$\hat{\varepsilon}_{i}=\frac{a_{i} \boldsymbol{x}-l_{i}}{\sigma_{i}}=\frac{\varepsilon_{i}}{\sigma_{i}}$.

The estimates of real errors are the corrections $v_{i}$ standard corrections $\hat{v}_{i}$ are defined:

$\hat{v}_{i}=\frac{v_{i}}{\sigma_{i}}$

It results in the estimate function:

$\rho\left(l_{i}, g_{i}(\boldsymbol{x})\right)=\rho\left(\hat{\varepsilon}_{i}\right)$.

Because $g_{i}(\overline{\boldsymbol{x}})=\boldsymbol{a}_{i}^{T} \overline{\boldsymbol{x}}$, the calculation of estimation can be adjusted to the form

$\sum_{i=1}^{n} \psi\left(\frac{a_{i} \boldsymbol{x}-l_{i}}{\sigma_{i}}\right) \frac{a_{i k}}{\sigma_{i}}=0, \sum_{i=1}^{n} \psi\left(\frac{v_{i}}{\sigma_{i}}\right) \frac{a_{i k}}{\sigma_{i}}=0$.

For the least squares method the following applies:

The density of probability of the normal distribution:

$$
\phi(x)=\frac{1}{\sigma \sqrt{2 \pi}} e^{-\frac{\{x-E(x)\}^{2}}{2 \sigma^{2}}}, \phi(\varepsilon)=\frac{1}{\sigma \sqrt{2 \pi}} e^{-\frac{\varepsilon^{2}}{2 \sigma^{2}}} .
$$

The function $\rho$ (without constants, after application of the logarithm): $\rho(\varepsilon)=\ln \left(e^{-\frac{1}{2}\left(\frac{\varepsilon}{\sigma}\right)^{2}}\right)=-\frac{1}{2}\left(\frac{\varepsilon}{\sigma}\right)^{2}$.

After derivation:

$\psi\left(\frac{\varepsilon}{\sigma}\right)=\frac{\partial\left(-\frac{1}{2}\left(\frac{\varepsilon}{\sigma}\right)^{2}\right)}{\partial \frac{\varepsilon}{\sigma}}=-\frac{1}{2} 2 \frac{\varepsilon}{\sigma}=-\frac{\varepsilon}{\sigma}$.

For minimization following must apply:

$\sum_{i=1}^{n}-\frac{\varepsilon_{i}}{\sigma_{i}} \frac{a_{i k}}{\sigma_{i}}=\sum_{i=1}^{n} \frac{\varepsilon_{i}}{\sigma_{i}} \frac{a_{i k}}{\sigma_{i}}=0$.

The real errors are not known, they will be replaced by their estimations - corrections. Solving the normal equations method of least squares is computationally simple. For using the calculation it is necessary to formally multiply the standardized corrections $\hat{v}_{i}$ by the correction coefficient $w_{i}$. Following must therefore apply:

$\sum_{i=1}^{n} \psi\left(\frac{v_{i}}{\sigma_{i}}\right) \frac{a_{i k}}{\sigma_{i}}=\sum_{i=1}^{n} w_{i} \frac{v_{i}}{\sigma_{i}} \frac{a_{i k}}{\sigma_{i}}=0$.

and then:

$\psi\left(\frac{v_{i}}{\sigma_{i}}\right) \frac{a_{i k}}{\sigma_{i}}=w_{i} \frac{v_{i}}{\sigma_{i}} \frac{a_{i k}}{\sigma_{i}}, \Rightarrow w_{i}=\psi\left(\frac{v_{i}}{\sigma_{i}}\right) / \frac{v_{i}}{\sigma_{i}}$.

The correction coefficient $w_{i}$ represents some weight of the measurement $l_{i}$, whose size is directly dependent on the size of standard corrections $\hat{v}_{i}, \mathrm{tj}$. $w_{i}=w_{i}\left(v_{i}, \sigma_{i}\right)$. After the introduction of the correction coefficient a modification can be done:

$\frac{1}{\sigma^{2}} \sum_{i=1}^{n} w_{i} v_{i} a_{i k}=0 \quad$ for $k \in\{1, \ldots, u\}$.

It is also possible to define the diagonal weight matrix $\boldsymbol{W}$ :

$\boldsymbol{W}=\operatorname{diag}\left(w_{1}, w_{2}, \ldots, w_{n}\right)$

and solve the normal equations in the form:

$\boldsymbol{A}^{T} \boldsymbol{W} \boldsymbol{A} \boldsymbol{x}=\boldsymbol{A}^{T} \boldsymbol{W} \boldsymbol{l}$.

Weights $w_{i}$ depends on the corrections $v_{i}$, i.e. on the estimate of the unknown $\boldsymbol{x}$. The estimate must be determined iteratively, the result of the least squares method can be used as a first approximation.

$\overline{\boldsymbol{x}}^{(0)}=\left(\boldsymbol{A}^{T} \boldsymbol{A}\right)^{-1} \boldsymbol{A}^{T} \boldsymbol{l}$,

$\overline{\boldsymbol{x}}^{(m+1)}=\left(\boldsymbol{A}^{\boldsymbol{T}} \boldsymbol{W}^{(m)} \boldsymbol{A}\right)^{-1} \boldsymbol{A}^{\boldsymbol{T}} \boldsymbol{W}^{(m)} \boldsymbol{l}$, 
$\boldsymbol{v}^{(m)}=A \overline{\boldsymbol{x}}^{(m)}-\boldsymbol{l}$.

In (Huber, 1964) the convergence of this calculation is proved.

As stated before, the robust adjustment methods are mostly based on the principle of maximum likelihood method and their basic property is (compared to in geodesy widely used the least squares method) high resistance against the influence (against) of outlying measurements. The principles and derivation of least squares and robust methods can be found in (Štroner and Hampacher, 2011). Most practically usable robust methods are based on adjusting of the weights in the calculation method of least squares (reweighting), such a calculation is then relatively easy. Methods are presented in (Štroner and Hampacher, 2011) too.

\section{PRINCIPLE OF THE PROCEDURE OF SHIFTS DETERMINATION - GENERAL CALCULATION PROCEDURE}

From the available epochs of measurement one has to be chosen, and that will be considered to be a basic one, and to which will be determined shifts related. Then there will be gradually carried out a transformation of all other epochs to the system of basic one, the transformation key will be determined by a robust adjustment. Here is a set of identical points as a set of measurements, whose errors due to the expected shift does not come from a normal probability distribution. Robust estimation method identifies those points that, in calculating the transformation parameters appear to be outlaying. These points will be from the set of identical points excluded. There will the obtained a sets of the identical points that will have various size. To calculate the final transformation key will be used only those points that are not identified as outliers and it will be calculated by the least squares method. Then determined transformation key will be used to transform all network points in the epoch to the system of the basic epoch. Differences between the coordinates of points in the basic epoch and transformed coordinates can be shifts, these differences are then tested by the statistical test. Basically the linear transformation between two epochs $i$ and $j$ is given by the equation:

$\boldsymbol{X}_{i}=\boldsymbol{R} \boldsymbol{X}_{j}+\boldsymbol{T}$,

where $\boldsymbol{X}_{\boldsymbol{i}}, \boldsymbol{X}_{\boldsymbol{j}}$ are vector of the coordinates, $\boldsymbol{M}$ matrix of the scale coefficients, $\boldsymbol{R}$ rotation matrix, $\boldsymbol{T}$ translation vector. The calculation procedure of iterative adjustment is based on the assembly of normal equations in the form:

$A^{T} P A d x=A^{T} P l^{\prime}$,

$d x=\left(A^{T} P A\right)^{-1} A^{T} P l^{\prime}$, where $\boldsymbol{A}$ is Jacobi matrix, $\boldsymbol{P}$ diagonal matrix of weights (on the diagonal are measurements' weights $P_{i i}=K / \sigma_{i}^{2}, K$ is chosen constant), $\boldsymbol{d} \boldsymbol{x}$ increment vector of unknowns, $\boldsymbol{l}$ ' vector of reduced measurements.

Robust weight change $\boldsymbol{W}$ must be then applied:

$d x=\left(A^{T} P W A\right)^{-1} A^{T} P W l^{\prime}$,

where robust weight change is determined by the equation:

$\boldsymbol{W}=\operatorname{diag}\left(w_{1}, w_{2}, \ldots, w_{n}\right) ; w_{i}=f\left(v_{i}, \sigma_{i}\right)$,

where corrections are determined by the equation:

$v=A d x-l^{\prime}$

with variance-covariance matrix:

$\boldsymbol{C o v}_{v}=K\left(\boldsymbol{P}^{-1}-\boldsymbol{A}\left(\boldsymbol{A}^{T} \boldsymbol{P} \boldsymbol{A}\right)^{-1} \boldsymbol{A}^{T}\right)$.

Robust changes are derived from the standard deviations of measurement and corrections obtained in adjustment. Various methods of calculating of the changes in weights can be used, derived on the basis of expected probability distribution of deviations from the normal distribution. Here it is worth mentioning Huber method (described in (Štroner and Hampacher, 2011)). When creating a robust estimator Huber came out from the normal random variable distribution. His solution is based on the replacement of the edge parts of the normal probability distribution by the Laplace distribution (a special form of the exponential distribution), which leads to greater probability of outlaying measurement on the distribution's edges.

For purposes of the analysis in this paper a $\mathrm{L}_{1}$ norm (the method minimizes the sum of absolute errors, and is also known as Least Absolute Deviations Method) was used, which, as a function of the probability distribution, uses directly Laplace distribution, which has in comparison to a normal distribution a greater probability of outlaying measurements occurrence. For homogeneous measurement (measurements with the same standard deviation) is a robust weight change given by the function

$w_{i}=1 /\left|v_{i}\right|$

and there is no need to know the standard deviation in this case. For nonhomogeneous measurement (measurements with the different standard deviations) is a robust weight change given by the function

$w_{i}=\sigma_{l} /\left|v_{i}\right|$.

The calculation is done iteratively, residuals used to calculate robust weights' changes are always used from a previous calculation. More to the calculation procedure in (Štroner and Hampacher, 2011).

If the residual exceeds the statistic criterion given by standard deviation of the residua and the 
reliability coefficient, the point is considered to be unstable.

\section{USE OF THE PROCEDURE ON THE DEFORMATIONS AND SHIFTS DETERMINATION OF THE PRAGUE CASTLE AREA}

Prague Castle is one of the most important historical, political and tourist areas of the Czech Republic, since 1918 also the seat of the President of the Czech Republic.

According to (Wikipedia, 2014), the Prague Castle complex was created by sequential additions and renovations of the settlement founded in the 9th century. With its dimensions of $570 \mathrm{~m}$ length and 128 $\mathrm{m}$ width it is one of the largest castle in the world. It is considered to be not only symbol of the city, but also the Czech statehood. Historic buildings located in the area are however affected by the aging process and the effects of changes in the surroundings. In order to predict further developments in this area, the long term periodic measurements for determining the stability of historic buildings in the area of the Prague Castle are carried out.

Geology in the area plays major role, according to (Záleský and Chamra, 2001) it was originally not complicated, but anthropogenic activities related to structural modifications of Hradčany hill during the last centuries made it considerably more complicated. The bedrock of the area has been reworked and expanded by the fills of different origin.

\subsection{GEODETIC MEASUREMENTS AT PRAGUE CASTLE}

Geodetic measurements were in the area of Prague Castle carried out in various range since its construction, but the periodic monitoring of selected historic buildings and slope stability is a matter of the last 15 years. There are changes monitored in tilt and relative height in individual buildings and areas. The first measuring was conducted by the Department of Special Geodesy in 1999, since then it is still ongoing and have been supported by several grants. The findings and conclusions of the measurements were summarized in (Procházka et al., 2011). These measurements were initially concentrated on the fault monitoring of individual buildings, and later connected via a network of reference points for both height measurements (precise levelling) and the position measurement. But because of this nonsystematic evolution of the measurement there are differences between epochs in configuration of the network and of monitored points, according to actual demand.

So far these measurements were evaluated only locally with respect to each building and it's stability without an overall view of the situation of possible shifts of individual parts of the surface of the Prague Castle area.

\subsection{THE HEIGHT ANALYSIS}

For the first analysis only height measurement was chosen. The reason for this decision is a very high and long-term achieved precision of $0.7 \mathrm{~mm} / \mathrm{km}$, also the high reliability and resistance of the method to systematic errors. Height measurements are almost entirely a matter of method levelling from the center with the addition of precise trigonometric method used to bridge the Jelení príkop (Deer Moat). Scheme of the performed measurements is in Figure 1. Measurements were conducted with use of the Zeiss $\mathrm{Ni} 007$ instrument, and in the last two epochs with use of the digital levelling instrument Trimble Dini 12T was used.

\subsubsection{NEW CALCULATION OF LEVELLING MEASUREMENTS EPOCHS}

Overall, there were 18 epochs re-processed and re-adjusted, all of them measured between years 2004 and 2012. Original intention of the measurements was not to carry out assessments, but monitoring of individual objects. Monitored objects changed during the years and in different epochs unequal sets of points were measured. Because of the relative solution of all monitoring, the measurement onto the stable points outside the Prague Castle area was not performed, and therefore none of the points can be considered to be stable.

Processing was made with regard to these facts in epochs by the least squares adjustment, in the GNU Gama software (gama-local, more in (Čepek, 2005)). As the measured values served averaged height differences measured back and forth. The a priori standard deviation was chosen to be $\sigma_{0}=0.7 \mathrm{~mm}$, because measurements were conducted predominantly by the Zeiss KoNi 007 instrument.

The results of the new adjustment are relative heights of points at each epoch. For further analysis, a standard deviation of height of one point is assumed to be $\sigma_{\mathrm{p}}=0.36 \mathrm{~mm}$ as the average of standard deviations of all points in all epochs. To identify the shifts between the epochs there was chosen an easy criterion, the height difference between the epochs should exceed $\Delta \mathrm{H}=\mathrm{u}_{\mathrm{p}} \cdot \sqrt{ } 2 \cdot \sigma_{\mathrm{p}}=1.0 \mathrm{~mm}$ for the $95 \%$ $\left(\mathrm{u}_{\mathrm{p}}=2\right.$, up is coefficient of the standard normal distribution, also known as t) or $1.3 \mathrm{~mm}$ for the probability of $99 \%\left(u_{p}=2.5\right)$.

\subsubsection{CALCULATION OF ROBUST ANALYSIS}

The results of the adjustment are relative heights of points at each epoch. It is not possible to consider any of the points to be stable, therefore the transformation with redundant measurements was chosen for the analysis and calculated with use of the robust estimation, which is highly resistant against the outlaying (here shifted) values.

There is only a one-dimensional transformation (only heights) needed, scale between the epochs does not change and therefore the transformation equation 


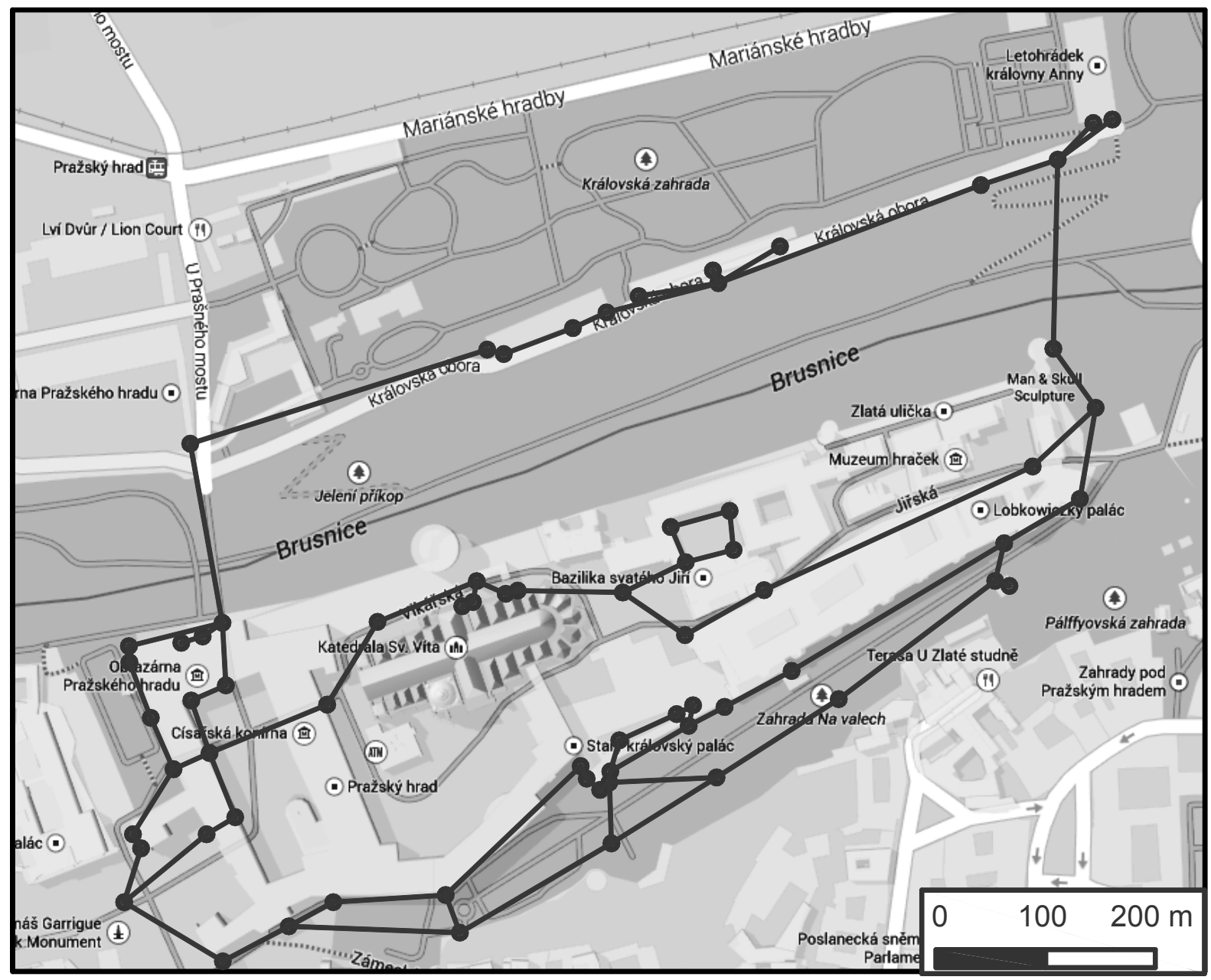

Fig. 1 Scheme of the height geodetic measurements in the Prague Castle area (underlying map from (Google Maps, 2014)).

for heights $\boldsymbol{H}$ between epochs $i$ and $j$ degrades as follows:

$$
H_{i}=H_{j}+T_{i, j}
$$

When calculating the relationship between the two epochs, it is determined only by height shift $T_{i, j}$, and there is the average height difference between the epochs:

$$
T_{i, j}=\frac{\sum_{k=1}^{n} H_{k, i}-H_{k, j}}{n} .
$$

Ideally, this shift will exactly suit to all points, though practically it does not, and therefore for every point $\mathrm{n}=1$.. $\mathrm{k}$ can be calculated corrections:

$$
v_{n}=H_{n, i}-\left(H_{n, j}+T_{i, j}\right) \text {. }
$$

These corrections contain a component of measurement inaccuracy, and if there was a height change, this influence too. Mean as a method corresponds with the least squares method, and in the case of outlaying measurements, here shifted points, fails to give proper results. For these reasons, it is advisable to use a robust method, which does not have such a property. The height difference between the two epochs is determined by an iterative calculation of the weighted average, where the weights are calculated on the basis of corrections from previous calculation ( $\mathrm{m}$-th iteration).

${ }^{(m+1)} T_{i, j}=\frac{\sum_{k=1}^{n}\left(H_{k, i}-H_{k, j}\right)^{(m)} w_{k}}{\sum_{k=1}^{n}{ }^{(m)} w_{k}}$.

The individual epochs were not measured at regular time intervals and also measured points changed, so the procedure has been used where at the selected epoch (namely 10) were gradually transfermed all the others. As the reference epoch was chosen epoch 10, because most points were measured in this epoch, both in initial and especially in the terminal epochs. The calculated shift $T_{i, j}$ is not important, significant are individual corrections signaling shifts of the point between the epochs. 


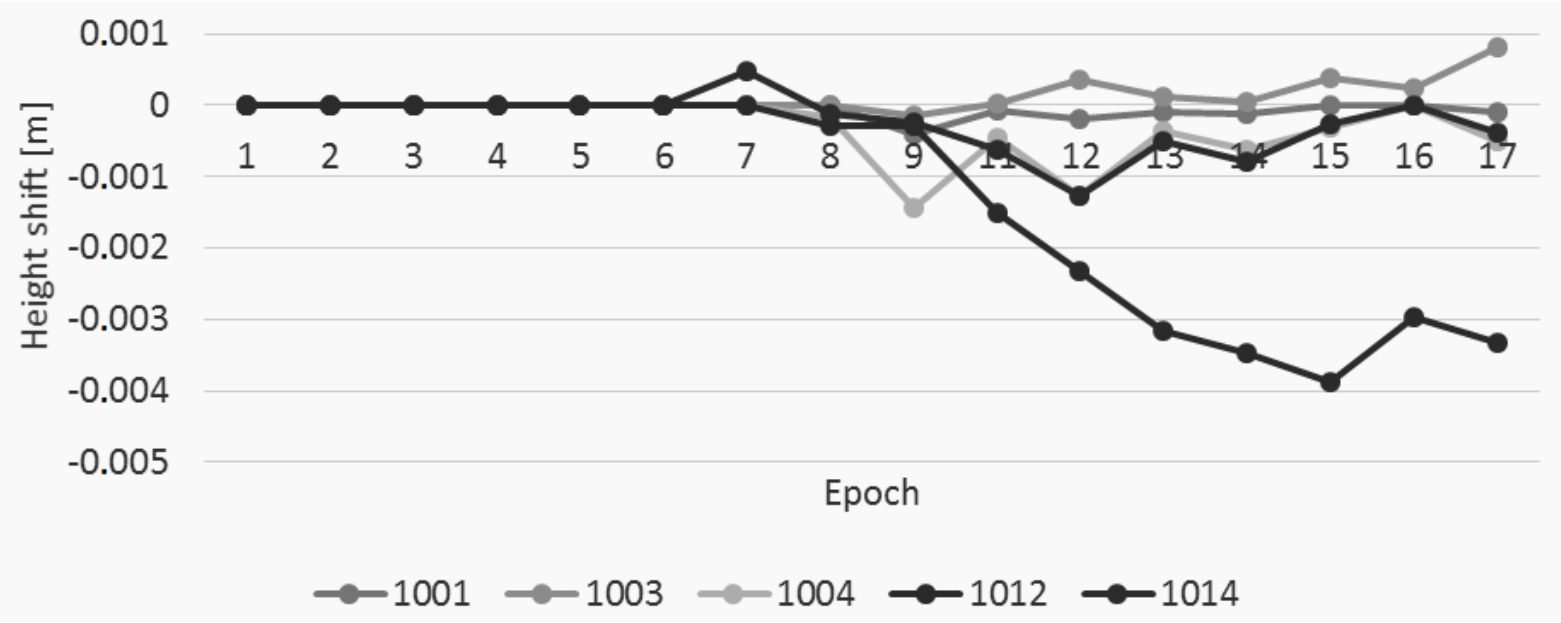

Fig. 2 Example of the relative points' shifts.

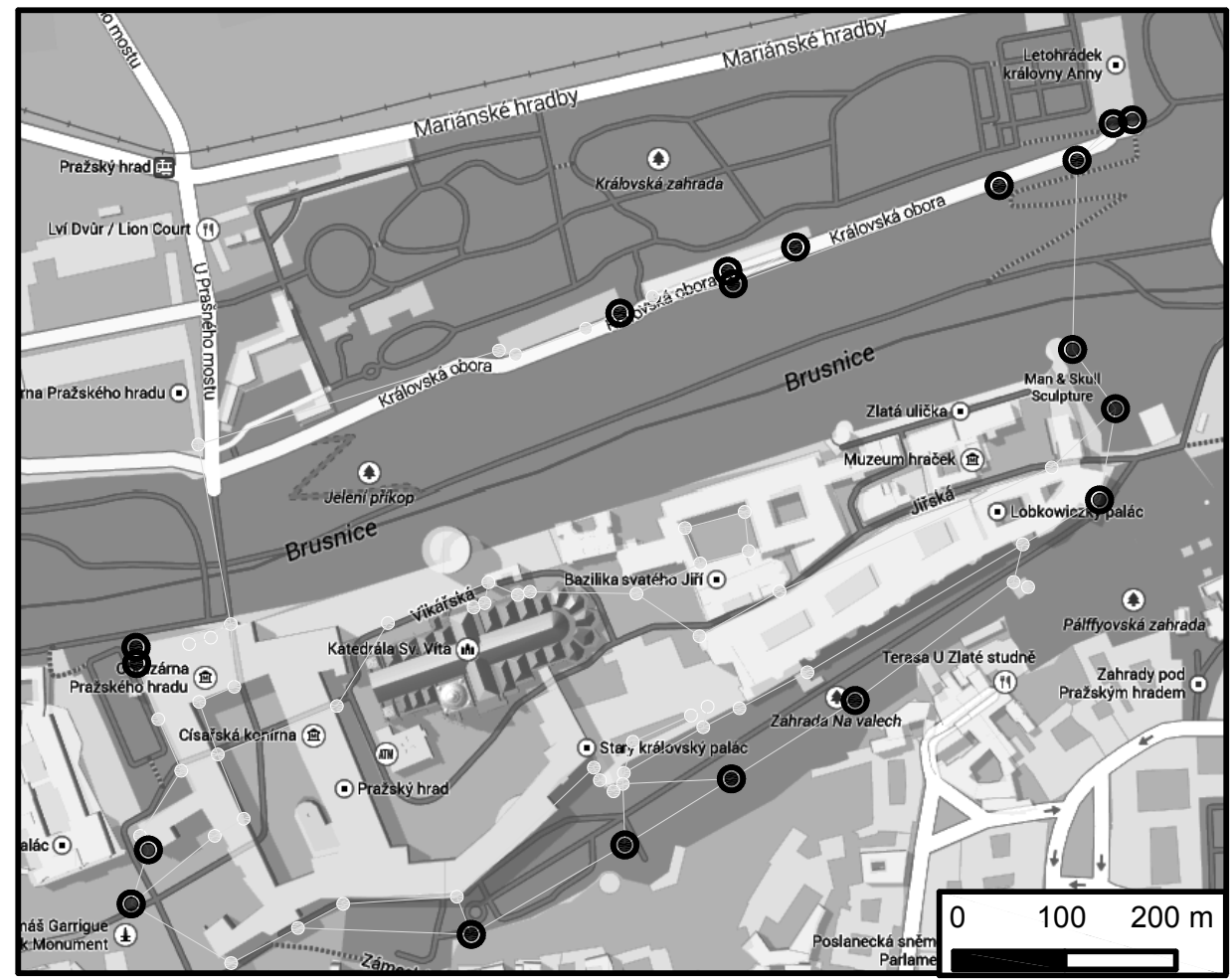

Fig. 3 Relative points shifts (underlying map from (Google Maps, 2014)).

\subsubsection{RESULTS}

The calculation results are determined corrections after the transformation, which can be interpreted as deviations of individual points from the common state from a common level.

When plotted on a graph, these corrections can give an idea of the shift of individual point between epochs. Because of the large number of points it is not possible to show all of it, an example is in Figure 2. Zero shift means, that point was not measured in the epoch. In Figure 3 there is an overview showing the points' shifts indicating some subareas' shifts. Characteristic shifting points are marked by the dark dot, light dots marks points considered to be stable.
As a result of the new evaluation of the deformation measurements at the Prague Castle, the scheme of relative height shift points was created, which is shown in Figure 2 and in Figure 3 is an overview showing the subareas' shifts. Points highlighted by the circle were identified as unstable, their height difference between the epochs exceeded $\Delta H=u_{p} \cdot \sqrt{ } 2 \cdot \sigma_{p}=1.0 \mathrm{~mm}$ for the $95 \%\left(u_{p}=2\right)$.

\subsection{THE POSITIONAL ANALYSIS}

The positional network consists of geotechnical boreholes, one deeply stabilized point (originally hydrogeological borehole) and selected geodetic points (Fig. 4). The geotechnical boreholes are usually 


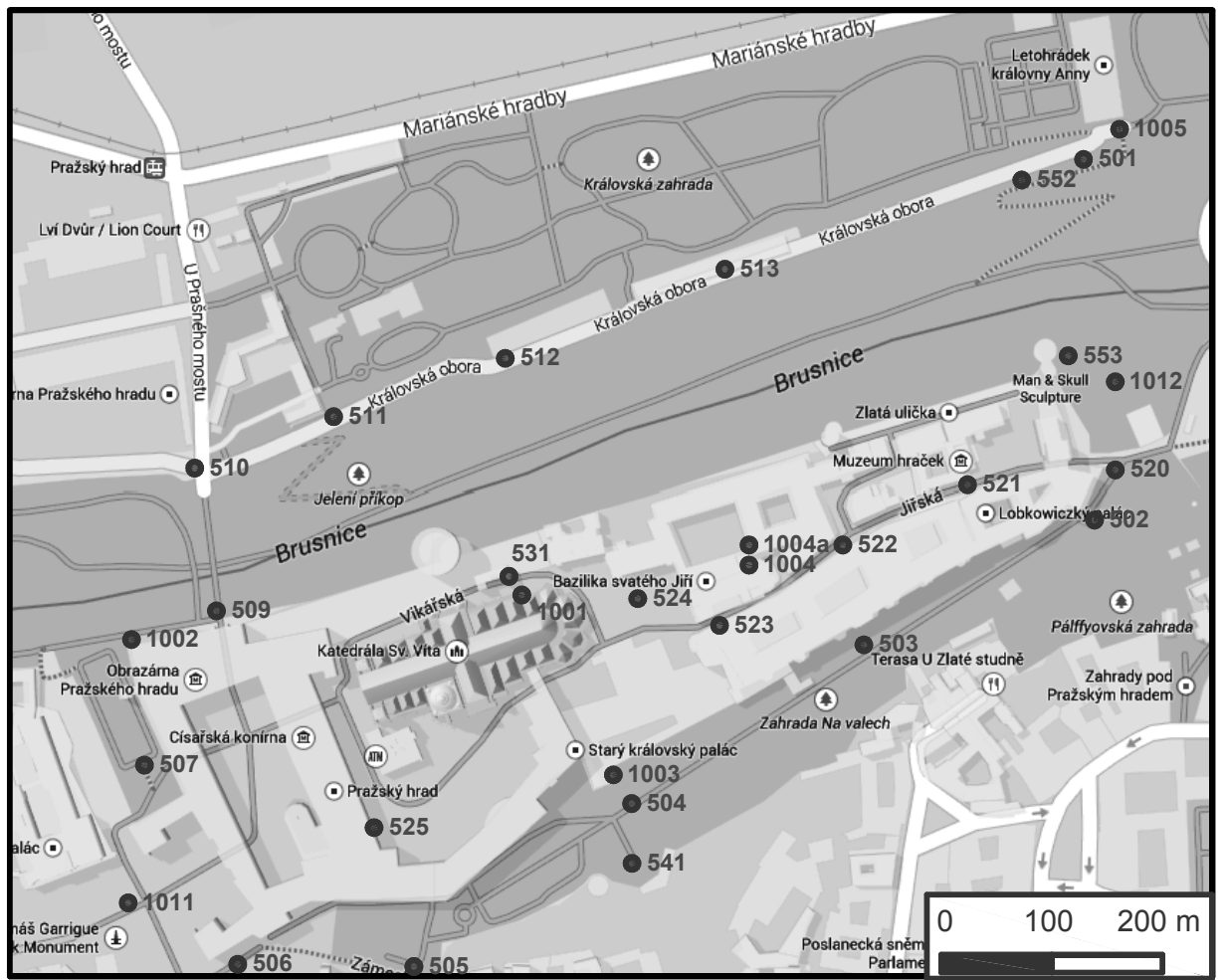

Fig. 4 Scheme of the measured points at Prague Castle (underlying map from (Google Maps, 2014)).

located near of significant buildings or near of those, which are at risk, such as the cathedral of St. Vitus, St. George, the Royal Summer Palace, etc. Positional network is determined by both classical terrestrial accurate polygons, and partly by GNSS (Global Navigation Satellite Systems) methods.

\subsubsection{A NEW ADJUSTMENT OF EPOCHS OF GEODETIC MEASUREMENTS}

As in case of the height network all the epochs of the positional measurement were readjusted. To adjust a positional network it is advisable to choose appropriate cartographic projection and convert all measured variables to it, it is advisable to choose such a projection, where the corrections of the direct measured values are minimize. The choice of cartographic projection can be further specified by the requirements, that there should not be lengths distortion practically. For this purpose was selected the Lambert Conformal Conic projection (LCC) with one parallel undistorted. This is a conformal projection of an ellipsoid to a plane through a cone in the normal position. Tangent cone touches the ellipsoid along selected parallel which is displayed as unbiased. This projection was chosen due to the elongated shape of the Prague Castle in nearly eastwest direction. Ellipsoid was used WGS-84. Since the linear distortion caused by the projection is due to the size of the network virtually imperceptible been directly measured length only reduced to null by the scale factor to a null horizon $m_{\text {red }}=0.99995298$ calculated from the average altitude of the area $\mathrm{H}=$ $300 \mathrm{~m}$. GNSS vectors were also transferred to this projection, including the transformation of the covariance matrix. GNSS measurement was realized at points 5013,1003,1005, 1011, 1012, in a minor number of epochs also at points 1002 or 524 .

Each epoch of the measurements was adjusted (by the least squares method) separately (together terrestrial and GNSS measurements) in the GNU Gama software (gama-local; (Čepek, 2005)). In the adjustment were considered the accuracies of measurement of both terrestrially measured values (through the standard deviations) and GNSS vectors (through their covariance matrix). The results of the adjustment are points, coordinates and their covariance matrix. The calculation was made as fixed on one point, which is probably stable and, in particular, to ensure that in all epochs the coordinates will not be too different for each point. One point fixation also does not affect neither the shape nor scale of the network. The absolute network location (absolute values of the coordinates) is not essential due to an examination of changes by the linear transformation. In total there were processed 8 epochs, from the year 2008 to year 2012 (two of each year, spring and autumn). In all epochs, was by the $\mathrm{chi}^{2}$ test confirmed the achievement of the estimated accuracy. The number of observations ranged from 104 (59 unknowns) to 232 (107 unknowns). For further processing of each epoch were used only coordinates and their covariance matrix. 


\subsubsection{PROCEDURE OF SHIFTS DETERMINATION}

From the available epochs of measurement one has to be chosen, and that will be considered to be a basic one, and to which will be determined shifts related. Then there will be gradually carried out a transformation of all other epochs to the system of basic one, the transformation key will be determined by a robust adjustment. Here is a set of identical points a set of measurements, whose errors due to the expected shift does not come from a normal probability distribution. Robust estimation method identifies those points that, in calculating the transformation parameters appear to be unstable. These points will be from the set of identical points excluded. There will be obtained sets of identical points that will have various size. To calculate the final transformation key will be used only those points that are identified as outliers and it will be calculated by the least squares method. Thus determined transformation key will be used to transform all network points in the epoch to the system of the basic epoch. Differences between the coordinates of points in the basic epoch and transformed coordinates can be shifts, these differences will be tested by the statistical test.

\subsubsection{CALCULATION PRINCIPLES OF TRANSFORMATION}

The equation of two-dimensional conformal linear transformation:

$$
\begin{aligned}
& \boldsymbol{X}=\boldsymbol{R}(\omega) \boldsymbol{x}+\boldsymbol{T}, \\
& \left(\begin{array}{l}
X \\
Y
\end{array}\right)=\left(\begin{array}{cc}
\cos (\omega) & -\sin (\omega) \\
\sin (\omega) & \cos (\omega)
\end{array}\right)\left(\begin{array}{l}
x \\
y
\end{array}\right)+\left(\begin{array}{l}
T_{x} \\
T_{y}
\end{array}\right),
\end{aligned}
$$

where $X$ is a vector of coordinates of the identical point in coordinate system of basic epoch, $x$ is a vector of coordinates of the identical point in coordinate system of transformed epoch, $\boldsymbol{R}(\omega)$ is the rotation matrix, $\omega$ is rotation angle and $\boldsymbol{T}$ is translation vector. In the case of a redundant number of identical points is transforming key calculated by the least squares method, as described above. The matrix $\boldsymbol{A}$ and vectors $\boldsymbol{l}$ ' and $\Phi$ (unknowns):

$$
\begin{aligned}
\boldsymbol{l}^{\prime} & =\left(\begin{array}{c}
x_{1}-X_{1} \\
y_{1}-Y_{1} \\
\vdots \\
y_{n}-Y_{n}
\end{array}\right), \boldsymbol{\Phi}=\left(\begin{array}{c}
T_{x} \\
T_{y} \\
\omega
\end{array}\right) . \\
\boldsymbol{A} & =\left(\begin{array}{ccc}
\frac{\partial X_{1}}{\partial T_{x}} & \frac{\partial X_{1}}{\partial T_{y}} & \frac{\partial X_{1}}{\partial \omega} \\
\frac{\partial Y_{1}}{\partial T_{x}} & \frac{\partial Y_{1}}{\partial T_{y}} & \frac{\partial Y_{1}}{\partial \omega} \\
\vdots & \vdots & \vdots \\
\frac{\partial X_{n}}{\partial T_{x}} & \frac{\partial X_{n}}{\partial T_{y}} & \frac{\partial X_{n}}{\partial \omega}
\end{array}\right)=\left(\begin{array}{ccc}
1 & 0 & \left(-x_{1} \sin \omega-y_{1} \cos \omega\right) \\
0 & 1 & \left(+x_{1} \cos \omega-y_{1} \sin \omega\right) \\
\vdots & \vdots & \vdots \\
0 & 1 & \left(+x_{n} \cos \omega-y_{n} \sin \omega\right)
\end{array}\right) .
\end{aligned}
$$

There are some possible variants of the calculation, which were tested. In the first variant of the calculation the precisions of the coordinates are considered to be equal and therefore all weights are equal to one. This variant is very simplified, and the results were in comparison with the other ones very different and therefore will not be described further.

In a second variant of the calculation were used different weights of measurements for each coordinate in the form of diagonal matrix only, because the use of full covariance matrices of point coordinates would require a much more complex complicate model of robust estimation with dependent observations. This problem is discussed e.g. in (Xu, 1989) or (Yang, 1994). Computational model, however, allows only be attribute the accuracy to one set of coordinates, i.e., the set, the coordinates are in adjustment the adjusted (vector $\boldsymbol{l}$ ). To consider the accuracy of coordinates in both two systems, both known covariance matrix has to be added. In our case, it is possible to do this step right, because both coordinate systems are rotated equally (practically), in adjustment were all epochs placed to the space the same way. As follows from the derivation of the transformation of the covariance matrix of the coordinates (e.g. in (Štroner and Hampacher, 2011), this act is dependent only on the mutual rotation of coordinate systems only.

The third and the most general used version is calculating the exact model transformation considering the accuracy of both systems. Measurements are now identical points in both systems in adjustment. Observation equations are therefore twofold:

$$
\begin{aligned}
& \left(\begin{array}{c}
{ }^{1} x_{i} \\
{ }^{1} y_{i}
\end{array}\right)=\left(\begin{array}{c}
X_{i} \\
Y_{i}
\end{array}\right), \\
& \left(\begin{array}{c}
{ }^{1} x_{i} \\
{ }^{1} y_{i}
\end{array}\right)=\left(\begin{array}{c}
T_{x} \\
T_{y}
\end{array}\right)+\boldsymbol{R}(\omega)\left(\begin{array}{c}
X_{i} \\
Y_{i}
\end{array}\right) .
\end{aligned}
$$

Coordinates $\left({ }^{1} x_{i},{ }^{1} y_{i}\right)$ are the coordinates in the system of the basic epoch into which it is transformed. Coordinates $\left({ }^{2} x_{i},{ }^{2} y_{i}\right)$ are the coordinates in the system of other epoch which is transformed. Coordinates $\left(X_{i}\right.$, $\left.Y_{i}\right)$ are along with elements of the transformation key unknown parameters. For the determination of weights will be used diagonal matrix again, but in this case it will contain the standard deviations different for all coordinates. This model is the most general from the presented ones.

\subsubsection{IDENTIFICATION AND TESTING OF DISPLACEMENT OF THE POINTS}

After calculating of the robust transformation are tested the residuals of the identical points through the limits $v_{M i}$ determined with use of the covariance matrix of the residuals for identical points using the formulas

$v_{x i}=v_{2 x i}-v_{1 x i}$, 


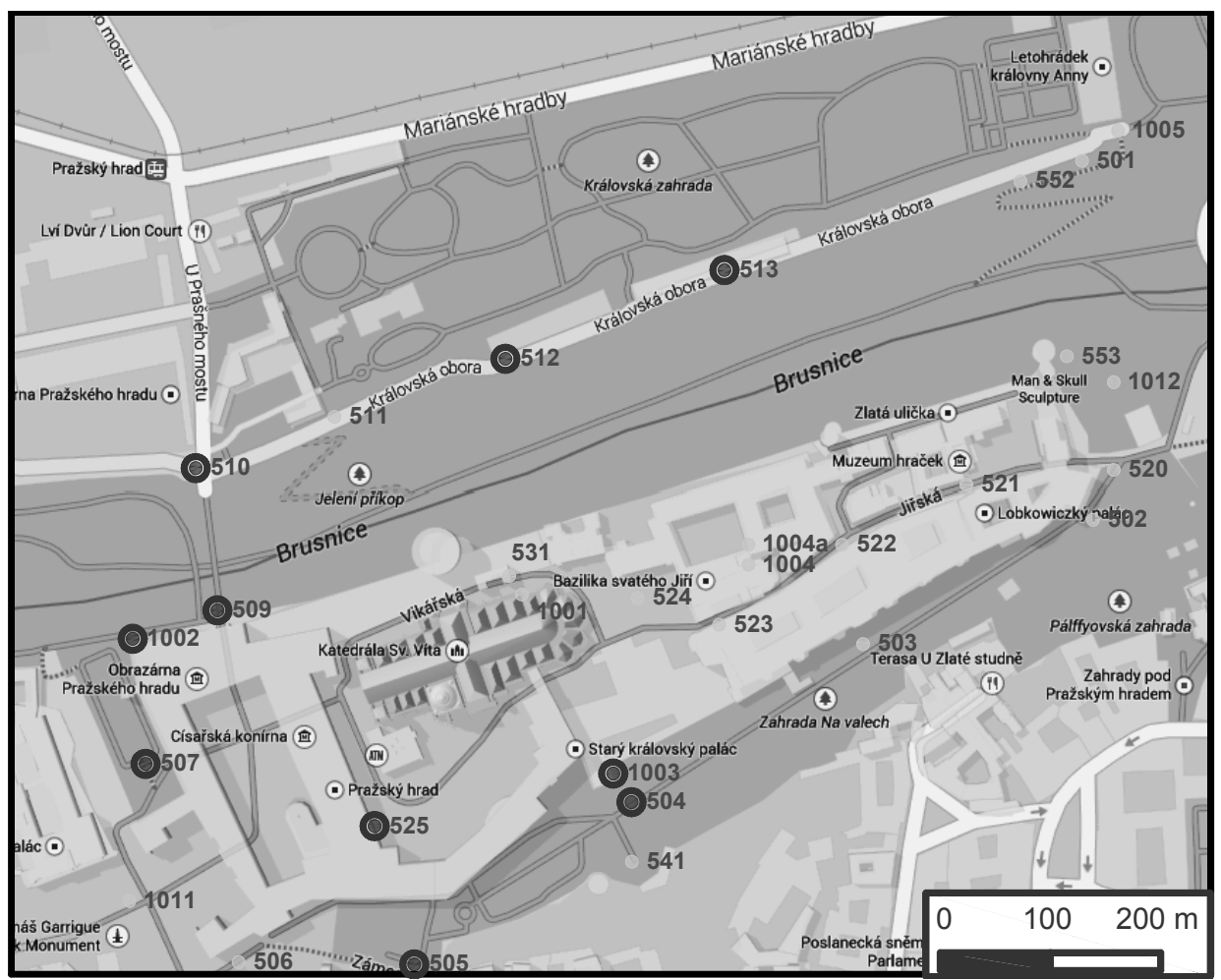

Fig. 5 Identified positionally unstable points (highlighted by the circle, underlying map from (Google Maps, 2014)).

$\operatorname{Cov}=K\left(\boldsymbol{P}^{-1}-\boldsymbol{A}\left(\boldsymbol{A}^{T} \boldsymbol{P} \boldsymbol{A}\right)^{-1} \boldsymbol{A}^{T}\right)$

$v_{M i}=\sqrt{\sigma_{v 2 x i}^{2}+\sigma_{v 1 x i}^{2}} u_{p}$,

where standard deviations are taken as an appropriate diagonal element from the covariance matrix $\boldsymbol{C o v}$ and $u_{p}$ is the critical value of the two-tailed test of normal distribution for probability $99 \%$. Thus identified potentially unstable points are not used for the final calculation of the transformation key. These potentially unstable points are transformed by the final transformation key and also the standard deviations of the resulting coordinates are calculated. Then the coordinate differences are calculated, a size of which is then tested by the limit $\Delta_{\mathrm{Mi}}$ of the difference calculated by the formula:

$\Delta_{M i}=\sqrt{\sigma_{x i}^{2}+\sigma_{x b a s}^{2}} u_{p}$,

where $\sigma_{\mathrm{xbas}}$ is standard deviation of the coordinate in the base epoch, $\sigma_{x i}$ in the transformed epoch. Unstable points were identified by this procedure, these are 504, 505, 507, 509, 510, 512, 513, 525, 1002, 1003. These points are marked in Figure 2.

\subsection{COMPARISON OF THE HEIGHT A POSITIONAL ANALYSIS}

Althrough the different of the displacement vector of each point were investigated in the paragraphs 4.2 and 4.3 , it is highly probable, that if the points or the areas are changing, it would be in both position and height. In Figure 6 there are shown the results of both height and positional shifts, and these are in some areas in good agreement, in some not.

The difference is probably due to the different sensitivity of methods given by the different accuracy (heights are determined with higher accuracy). Positional and height stabilization is different and in some cases the shift of the point could be only a shift of the surface stabilization. According to the result of both methods the central area is stable. Identified unstable points lie at the edges of the raised area of Prague Castle, and it also well corresponds with the information given in the introduction, that the bedrock of the area has been reworked and expanded by the fills of different origin.

\section{CONCLUSIONS}

A new methodology was described for evaluating of the deformation measurements at the Prague Castle, it involves the use of linear transformation and a robust estimation, namely the L1 norm. The methodology was successfully used on the new evaluation of the deformation measurements at the Prague Castle in two different types of measurement (first for a height network and second for a positioning network). The process was divided in two parts, because the measurements were done separately and also the stabilization of the points is 


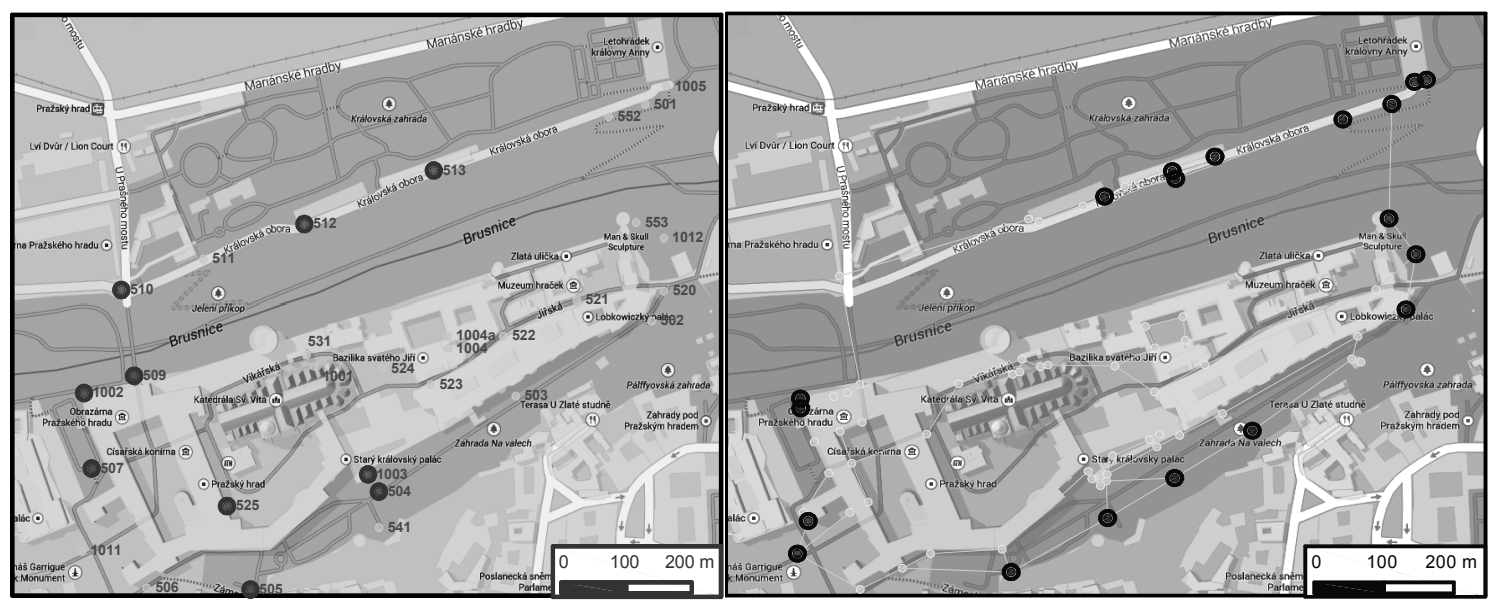

Fig. 6 Identified points unstable in position on the left (highlighted by the circle), on the right side the result from evaluation of the height measurement (underlying map from (Google Maps, 2014)).

physically separated. As a result of the evaluation the scheme of relative shifts of points was created.

Both height and positional results are in general mutually consistent and also consistent with observed phenomena in the field. According to the result of both methods the central area is stable. Identified unstable points lie at the edges of the raised area of Prague Castle, and it also well corresponds with the information given in the introduction, that the bedrock of the area has been reworked and expanded by the fills of different origin.

However, there must be clearly stated that the evaluation determines only relative changes in the monitored area, not absolute changes. E.g. in the case of displacement of the whole area it will not reveal any change.

\section{ACKNOWLEDGEMENTS}

The article was written with support of the internal grant of Czech Technical University in Prague SGS14 "Optimization of acquisition and processing of 3D data for purpose of engineering surveying“.

\section{REFERENCES}

Colesanti, C and Wasowski, J.: 2006, Investigating landslides with space-borne synthetic aperture radar (SAR) interferometry. Engineering Geology, 88, 3-4, 173-199. DOI:10.1016/j.enggeo.2006.09.013

Čepek, A.: 2005, GNU Gama 1.9 - Adjustment in geodetic networks. Edition 0.19, 2005.

Darby, D.J. and Williams, R.O.: 1991, A new geodetic estimate of deformation in the central volcanic region of the North Island, New-Zealand. New Zealand Journal of Geology and Geophysics, 34, 2, 127-136. DOI: $10.1080 / 00288306.1991 .9514450$

Dunning, S.A., Massey, C.I. and Rosser, N.J.: 2009, Structural and geomorphological features of landslides in the Bhutan Himalaya derived from Terrestrial Laser Scanning. Geomorphology, 103, 1, 17-29. DOI:10.1016/j.geomorph.2008.04.013
Gokalp, E. and Tasci, L.: 2009, Deformation monitoring by GPS at embankment dams and deformation analysis. Survey Review, 41, 311, 86-102.

DOI: $10.1179 / 003962608 X 390021$

Google Maps: http://maps.google.com, cit. 5.11.2014.

Hamouz, J., Braun, J. Urban, R. Štroner, M. and Vráblík, L.: 2014, Monitoring and evaluation of prestressed concrete element using photogrammetric methods. In: 14th International Multidisciplinary Scientific Geoconference SGEM 2014, Conference Proceedings vol. III. Sofia: STEF92 Technology Ltd., 231-238.

Hampel, F.R., Ronchetti, E.M., Rousseeuw, P.R. and Stahl, W.A.: 1986, Robust statistics. Wiley, New York.

Hruska, J. and Hubatka, F.: 2000, Landslides investigation and monitoring by a high-performance ground penetrating radar system. In: GPR 2000: Proceedings of the Eighth International Conference on Ground Penetrating Radar. Proceedings of the Society of Photo-Optical Instrumentation Engineers (SPIE). Univ Queensland, Gold Coast, Australia, May 23-26, 2000. 4084, 688-693. DOI:10.1117/12.383500

Huber, P.J.: 1964, Robust estimation of a location parameter. Annals of Mathematical Statistics, 35, 73101.

Huber, P.J.: 1981, Robust statistics. New York: John Wiley $\&$ Sons.

Koch, K.R.: 1999, Parameter estimation and hypothesis testing in linear models. Springer Verlag, Berlin Heidelberg New York.

Koska, B., Křemen, T., Pospíšil, J., Kyrinovič, P. and Haličková, J.: 2008, Monitoring of lock chamber dynamic deformation. In: Proceeding of Measuring the Changes - 13th FIG Symposium on Deformation Measurements and Analysis and 4th IAG Symposium on Geodesy for Geotechnical and Structural Engineering. LNEC, Lisbon.

Pavelka, K. and Reznicek, J.: 2010, Photogrammetrical measuring of the dynamical deformation of the joint and the column web panel at elevated temperature. In: 31st Asian Conference on Remote Sensing 2010, 1-5 November 2010, Hanoi, Vietnam.

Prague Castle, Wikipedia, cit. 27.10.2014. http://en.wikipedia.org/wiki/Prague Castle. 
Pospíšil, L.; Roštínský, P.; Švábenský, O.; Weigel, J. and Witiska, M.: 2012, Active tectonics in the eastern margin of the Bohemian massif - based on the geophysical, geomorphological and GPS data, Acta Geodyn. Geomater., 9, 3 (167), 315-329.

Procházka, J., Záleský, J., Jiřikovský, T. and Salák, J.: 2010, Long-term stability monitoring in the Prague Castle area. Acta Geodyn. Geomater., 7, 4 (160), 411-429.

Procházka, J., Jiřikovský, T., Záleský, J. et al.: 2011, Stability of historic buildings. Prague, CTU Publishing House, 229 pp., (in Czech).

Rueger, J.M.: 2006, Overview of geodetic deformation measurements of dams. Proceedings of the Ancold 2006 Conference, Sydney, Australia.

Sabová, J. and Pukanská, K.: 2007, Deformation measurement project. Acta Montanistica Slovaca, 12, 3, 516-519. (in Slovak).

Štroner, M. and Hampacher, M.: 2011, Processing and Analysis of Measurements in Engineering Surveying. Prague, CTU Publishing House, 313 pp., (in Czech).

Teskey, W.F., Lovse, J.W. and Al Hanbali, N.N.: 1996, Deformation, alignment, and vibration in large turbine-generator set. Journal of Surveying Engineering-Asce, 122, 2, 65-79. DOI: 10.1061/(ASCE)0733-9453(1996)122:2(65)

Třasák, P. and Štroner, M.: 2014, Outlier detection efficiency in the high precision geodetic network adjustment. Acta Geodaetica et Geophysica, 49, 2. DOI: $10.1007 / \mathrm{s} 40328-014-0045-9$

$\mathrm{Xu}$, P.: 1989. On robust estimation with correlated observations. Bulletin Geodesique. Berlin, Springer, 63, No. 3, 237-252. DOI: 10.1007/BF02520474

Yang, Y.: 1994, Robust estimation for dependent observations. Manuscripta Geodaetica. Berlin, Springer, 19, No. 1, 10-17.

Záleský, J. and Chamra, S.: 2001, Technical conditions of historic buildings monitoring project. In: Optimalizácia geotechnických konštrukcií, 18. - 19. September 2001, SvF STU Bratislava, Slovak Republic, 337-341, (in Czech). 